\title{
The inflammasome in fibromyalgia and CRPS: a microglial hypothesis?
}

Mario D. Cordero

I read with interest the Review article by Littlejohn (Neurogenic neuroinflammation in fibromyalgia and complex regional pain syndrome. Nat. Rev. Rheumatol. doi:10.1038/nrrheum.2015.100; 2015). ${ }^{1}$ This very informative paper describes the contribution of neurogenic neuroinflammation to the pathophysiology of fibromyalgia and complex regional pain syndrome (CRPS). However, I was surprised that a discussion of the inflammasome was not included in this Review.

The inflammasome is a protein complex comprising an intracellular sensor, typically a NOD-like receptor (NLR), the precursor procaspase- 1 and the adaptor protein ASC. Inflammasome activation leads to the maturation of caspase-1 and the processing of its substrates, IL- $1 \beta$ and IL-18. ${ }^{2}$ Among the NLRs, NLRP 3 is activated by the most diverse array of danger signals. Unexpectedly, the inflammasome has recently emerged as a sensor of metabolic derangement and stress. Indeed, it is implicated in the development of major human diseases such as gout, type 2 diabetes (and obesityinduced insulin resistance), Alzheimer disease and cancer, as well as pathologies such as asbestosis. ${ }^{3}$ The inflammasome also plays a part in several rheumatic disorders. ${ }^{4}$ With respect to fibromyalgia and CRPS, my research group has shown that the NLRP3 inflammasome participates in the pathophysiology of fibromyalgia in patients and animal models, and has studied the effect of NLRP3 inhibition., ${ }^{5,6}$ Previous studies in fibromyalgia showed that the $M E F V$ gene (which encodes pyrin, a major regulator of the inflammasome) is implicated in the control of caspase- 1 activation and in dysregulation of IL- $1 \beta$ secretion. ${ }^{7}$ Moreover, in CRPS, the NALP1 inflammasome is involved in cytokine production and nociception, ${ }^{8}$ and nociceptive sensitization can be induced by NALP1-dependent and IL-1 $\beta$-dependent production of neuropeptides. ${ }^{9}$
Dysregulated inflammasome activation, via altered innate immune cell function, has been implicated in the establishment of chronic, low-grade inflammation. ${ }^{2-4}$ This dysregulation is mediated by several immune cell types, including macrophages and dendritic cells. ${ }^{10}$ Similarly, excessive activation of innate immune responses in the central nervous system (CNS) can promote pathology through off-target actions leading to inflammasome activation. ${ }^{11}$ Interestingly, inflammasome activation in the CNS primarily occurs in microglia and macrophages. ${ }^{11}$ Microglia have been highly studied as key contributors to pathological and chronic pain mechanisms, ${ }^{12}$ and are involved in hyperalgesia and allodynia in both fibromyalgia and chronic fatigue syndrome, as well as pain in CRPS. ${ }^{13,14}$ Microglial inflammasome activation promotes the recruitment of peripheral innate immune cells (macrophages) and adaptive immune cells ( $\mathrm{T}$ cells and B cells), as well as further activating nearby glial cells, which could be responsible for the peripheral effects proposed by Littlejohn. ${ }^{1}$ Of note, this Review article mentions that downregulation of activated glial cells is a potential strategy for targeting neurogenic neuroinflammation in fibromyalgia and CRPS, so an inflammasome-induced microglial activation hypothesis could be plausible.

In conclusion, I agree with the interesting proposal of Littlejohn; however, this proposed inflammasome-microglial activation mechanism might be an additional link in fibromyalgia, CRPS and chronic fatigue syndrome that could offer novel therapeutic targets. In this respect, inhibition of NLRP3 is a promising approach. ${ }^{15}$

Instituto de Biomedicina de Sevilla (IBiS), Universidad de Sevilla, Avda Manuel Siurot s/n, 41013 Seville, Spain.

mdcormor@us.es

\section{Acknowledgements}

The author's research work is supported by Grupo de Investigacion Junta de Andalucia CTS113, Consejería de Salud, Junta de Andalucia (PI-0036-2014).
Competing interests

The author declares no competing interests.

1. Littlejohn, G. Neurogenic neuroinflammation in fibromyalgia and complex regional pain syndrome. Nat. Rev. Rheumatol. http:// dx.doi.org/10.1038/nrrheum.2015.100.

2. Leemans, J. C., Cassel, S. L. \& Sutterwala, F. S. Sensing damage by the NLRP3 inflammasome. Immunol. Rev. 243, 152-162 (2011).

3. Guo, H., Callaway, J. B. \& Ting, J. P. Inflammasomes: mechanism of action, role in disease, and therapeutics. Nat. Med. 21, 677-687 (2015).

4. So, A., Ives, A., Joosten, L. A. \& Busso, N. Targeting inflammasomes in rheumatic diseases. Nat. Rev. Rheumatol. 9, 391-399 (2013).

5. Cordero, M. D. et al. NLRP3 inflammasome is activated in fibromyalgia: the effect of coenzyme $\mathrm{Q}_{10}$. Antioxid. Redox Signal. 20, 1169-1180 (2014).

6. Bullon, P. et al. AMPK phosphorylation modulates pain by activation of NLRP3inflammasome. Antioxid. Redox Signal. http:// dx.doi.org/10.1089/ars.2014.6120.

7. Feng, J. et al. Missense mutations in the MEFV gene are associated with fibromyalgia syndrome and correlate with elevated IL-1 $\beta$ plasma levels. PLoS ONE 4, e8480 (2009).

8. Li, W. W. et al. The NALP1 inflammasome controls cytokine production and nociception in a rat fracture model of complex regional pain syndrome. Pain 147, 277-286 (2009).

9. Shi, X. et al. Neuropeptides contribute to peripheral nociceptive sensitization by regulating interleukin- $1 \beta$ production in keratinocytes. Anesth. Analg. 113, 175-183 (2011).

10. So, A. \& Busso, N. The concept of the inflammasome and its rheumatologic implications. Joint Bone Spine 81, 398-402 (2014).

11. Walsh, J. G., Muruve, D. A. \& Power, C. Inflammasomes in the CNS. Nat. Rev. Neurosci. 15, 84-97 (2014).

12. Milligan, E. D. \& Watkins, L. R. Pathological and protective roles of glia in chronic pain. Nat. Rev. Neurosci. 10, 23-36 (2009).

13. Yasui, M. et al. A chronic fatigue syndrome model demonstrates mechanical allodynia and muscular hyperalgesia via spinal microglial activation. Glia 62, 1407-1417 (2014).

14. Schwartzman, R. J., Alexander, G. M. \& Grothusen, J. Pathophysiology of complex regional pain syndrome. Expert Rev. Neurother. 6, 669-681 (2006).

15. Onuora, S. Inflammation. Potent small molecule extinguishes the NLRP3 inflammasome. Nat. Rev. Rheumatol. 11, 198 (2015). 